\title{
Extraction and Solution of the Gyroplane Trim Equations
}

\author{
A. Osaji ${ }^{*}, 1 \S$ and H. Farrokhfal ${ }^{2}$ \\ ${ }^{1}$ Department of Aerospace Engineering, Sharif University of Technology, Tehran, Iran \\ ${ }^{\S}$ Present Address: No 71, Farhang Avenue, Roodsar, Guilan, Iran \\ ${ }^{2}$ Department of Mechanical Engineering, Isfahan University of Technology, Isfahan, Iran
}

\begin{abstract}
Extraction and solution of the gyroplane trim equations are the goal of this paper. At first according to forces and moments acting on gyroplane and the different parts of gyroplane, the six equations of the rigid body will be extracted. Because of the trim conditions, angular velocity and linear acceleration are considered to be zero. Then the energy balance equation will be derived by modeling forces and velocities. The energy balance equation shows the advancing efficient power, which is derived from the engine, is equal to all depleted powers (induced power, profile power, main rotor power, parasite power, climb power) by the gyroplane. Then by modeling non-dimensional velocities in the parallel and perpendicular directions of the main rotor reference plane, the main rotor momentum equation will be extracted. The forces of each different parts of the gyroplane will be derived according to the trim control parameters in the body axes. They will be assigned into the rigid body equations of motion. By solving the rigid body, energy balance and main rotor momentum equations, for several flight scenarios, the pilot trim control parameters (containing thrust, angle of attack for reference plane, pilot blade pitch angle and Rudder angle) will be obtained.
\end{abstract}

\section{INTRODUCTION}

The gyroplane structure is a combination of the airplane and helicopter. It has a propeller engine as airplane. For more stability, the engine is placed in the back of the gyroplane center of mass. It has a main rotor as helicopter but the rotor is not jointed to any engine and does autorotation. When the pilot brings up the reference plane, the rotor takes up wash. Because of drag force differential between two blades, the rotor auto rotates and produces lift and drag. The main rotor rotates freely and produces much lift. Therefore the gyroplane band length is short (about 30 meters). It has invented before helicopter. After the helicopter invention, usage of the gyroplane significantly becomes decreased. It is safer than helicopter when the engine fails because of its autorotation. However it can not flight vertically nor does hovering flight as the helicopter. The gyroplane price is cheaper than the helicopter price. At now people in many countries in the world have gone back to use gyroplane because of these advantages [1].

\section{THEORETICAL DISCUSSIONS AND RESULTS}

By considering a control volume around the gyroplane, from the Reynolds Ronald's transportation formula (transportation from system to control volume) the conservative rule of linear, angular momentum, mass and energy can be derived for it. The conservative rule of the linear momentum gives three equations of motion in three perpendicular directions. The conservative rule of angular momentum gives three angular equations of motion around

*Address correspondence to this author at the Department of Aerospace Engineering, Sharif University of Technology, Tehran, Iran; Tel: $+98-142$ 6211577; E-mail: aliosaji@gmail.com the three perpendicular directions. From the conservative rule of mass the continuous equation can be derived and from the conservative rule of the energy the scalar equation for the produced and consumed powers can be derived. In addition to these equations three kinematics equations and three navigation equations can be derived from the Euler angles. If the gyroplane be surrounded by a control volume, all of these equations can be derived for it. Because of trim conditions, its acceleration and angular velocity should be considered to be zero. Therefore the six kinematics and navigation equations will be cancelled. Because its mass is considered to be constant, the continuous mass equation will be cancelled. Therefore following equations will be derived for the gyroplane [2].

$$
\begin{aligned}
& \mathbf{F}_{A}+\mathbf{F}_{T}+\mathbf{F}_{W}=0 \\
& \mathbf{M}_{A}+\mathbf{M}_{T}=0 \\
& \sum E=0
\end{aligned}
$$

where $\mathbf{F}$ and $\mathbf{M}$ represent the force and momentum vector and the subscripts $A, T$ and $W$ represent aerodynamics, thrust and weight. $E$ represents energy.

The main rotor is not rigid respect to the other sections of the gyroplane therefore to consider its effect; the blade momentum equation will be derived separately.

The subscripts $(M, P, H, V, F)$ represent main rotor, propeller engine, horizontal tail, vertical tail, and fuselage.

According to Figs. (1-3) six equations of the rigid body will be as equations (1) to (6) $[3,4]$

$$
\begin{aligned}
& X_{M}+X_{P}+X_{H}+X_{V}+X_{F}=W \sin \left(\theta_{b}\right) \\
& Y_{M}+Y_{P}+Y_{V}+Y_{F}=-W \sin \left(\phi_{b}\right)
\end{aligned}
$$




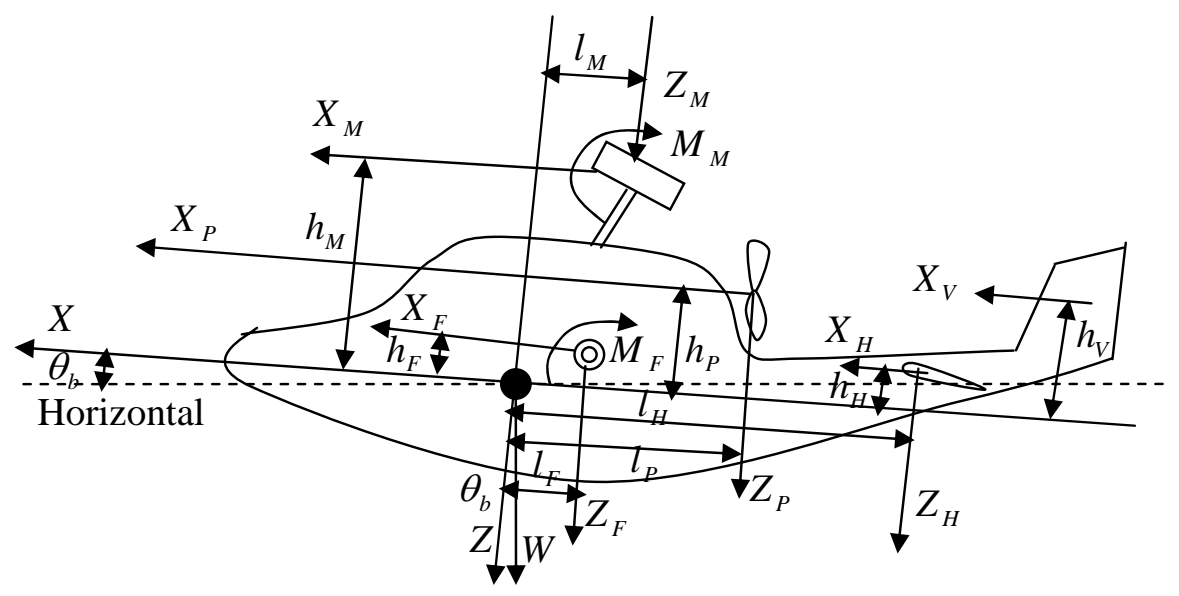

Fig. (1). Side view for showing gyroplane forces and moments.

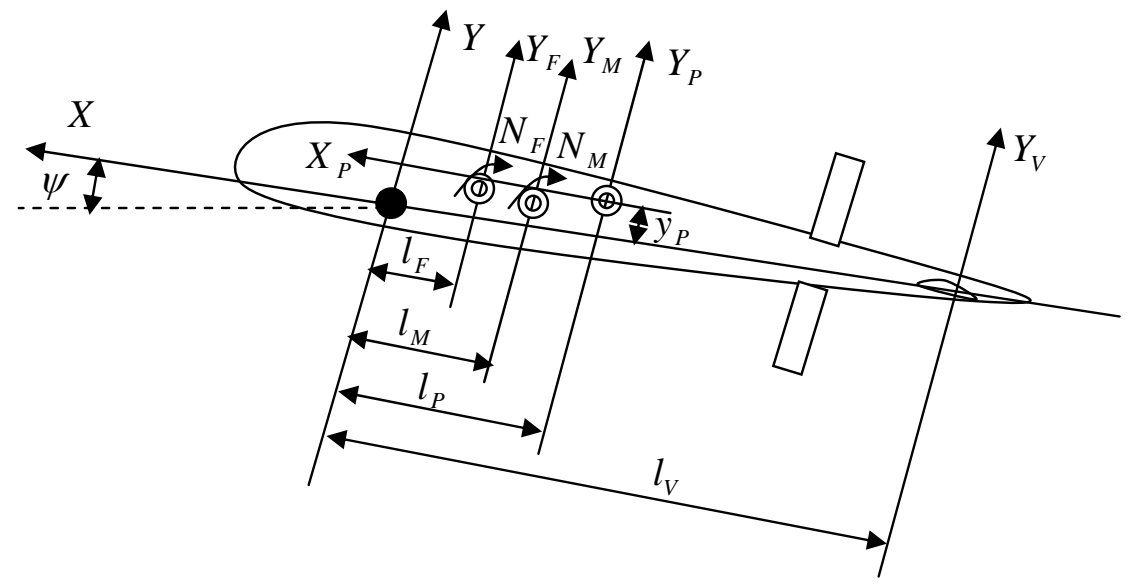

Fig. (2). Top view for showing gyroplane forces and moments.

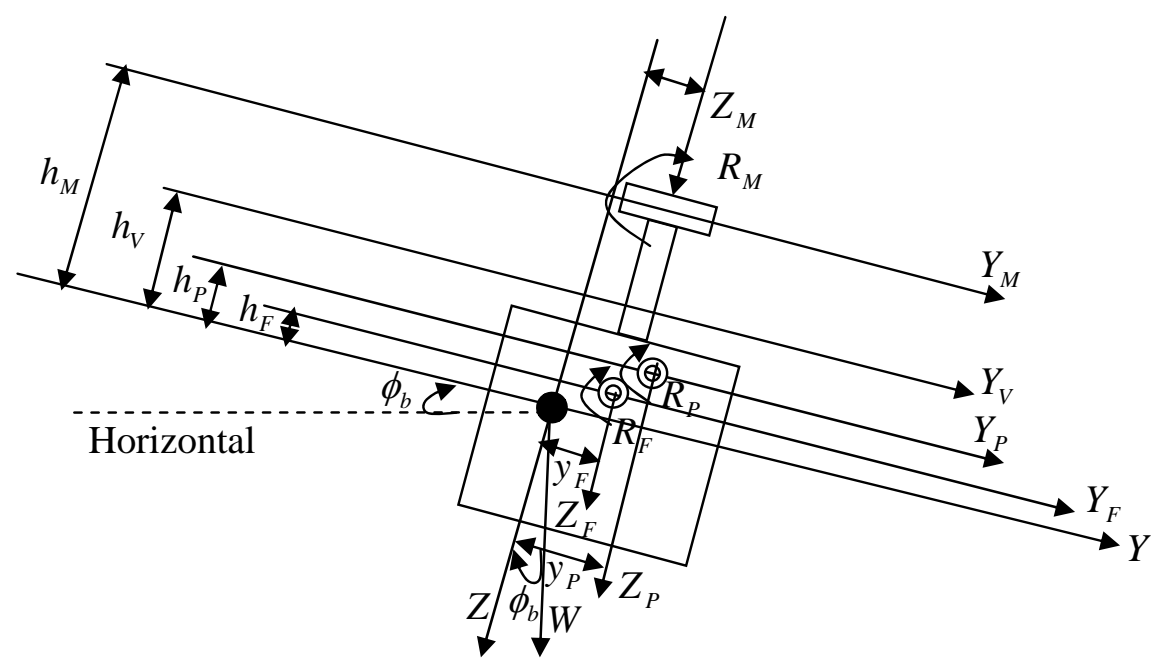

Fig. (3). Rear view for showing gyroplane forces and moments.

$(R, M, N)$ In order represent roll, pitch and yaw moments around the body axes.

$(X, Y, N)$ Represent forces in the longitudinal, lateral and vertical directions of the body axes.

$(l, y, h)$ Represent longitudinal, lateral and vertical distance from ths nter of mass. The center of body axes is in the center of mass. 


$$
\begin{aligned}
& Z_{M}+Z_{P}+Z_{H}+Z_{V}+Z_{F}=-W \cos \left(\theta_{b}\right) \\
& (R+Y h+Z y)_{M}+(R+Y h+Z y)_{P}+(Z y)_{H} \\
& +(Y h)_{V}+(R+Y h+Z y)_{F}=0 \\
& (M-X h+Z l)_{M}+(-X h+Z l)_{P}+(-X h+Z l)_{H}+ \\
& (-X h)_{V}+(M-X h+Z l)_{F}=0 \\
& (N-Y l-X y)_{M}+(-Y l-X y)_{P}+(-X y)_{H}+ \\
& (-Y l-X y)_{V}+(N-Y l-X y)_{F}=0
\end{aligned}
$$

Consider $\Psi=\beta_{b}$ where $\beta_{b}$ is side slip and $\theta_{b}, \varnothing_{b}$ are body pitch and roll angles. Suppose $\varnothing_{b}=0$,

$M_{V}=N_{H}=R_{V}=R_{H}=R_{F}=0$ and $W$ is the gyroplane gravity weight.

The efficient power given to the gyroplane system which is taken from the engine is equal to $T_{P} . V$. Where $V$ is the gyroplane advance velocity. In the other word some parts of the engine power will be used to conquer on profile, induced power of the propeller and friction power of the engine axes.

The gyroplane consumed powers are induced power $\left(P_{i}\right)$, profile power $\left(P_{\text {prof }}\right)$, main rotor friction power $\left(P_{f M}\right)$, parasite power $\left(P_{\text {paraz }}\right)$, climb power $\left(P_{c}\right)$. Therefore the energy balance equation will be as:

$T_{P} . V=\eta_{P} P_{P}=P_{i}+P_{\text {prof }}+P_{\text {paraz }}+P_{f M}+P_{c}$, where $\eta_{P}$ is the engine power efficiency coefficient and $\eta_{P}<1$.

At now these powers can be derived according to power coefficients:

$$
P_{P}=C_{P_{P}} \rho \pi R_{P}^{2}\left(R_{p} \omega\right)^{3} \text { and } P_{f M}=C_{P_{M}} \rho \pi R^{2}(R \Omega)^{3} \text { Where } \Omega, \omega
$$

are the main rotor and propeller rpm and $R, R_{P}$ are the main rotor and propeller radius.

If the two sides of the energy balance equation be divided by $\rho \pi R^{2}(R \Omega)^{3}$, it changes to: $C_{P_{P}} \eta=C_{P_{i}}+C_{P_{\text {prof }}}+C_{P_{\text {Paraz }}}+C_{P_{P M}}+C_{p_{C}}$ where $\eta=\eta_{P} \frac{\rho \pi R_{P}{ }^{2}\left(R_{p} \omega\right)^{3}}{\rho \pi R^{2}(R \Omega)^{3}}$, therefore $T_{P} . V=\rho \pi R^{2}(R \Omega)^{3}\left(C_{P_{i}}+C_{P_{\text {prof }}}+C_{P_{\text {Paraz }}}+C_{P_{\text {PM }}}+C_{p_{C}}\right)$.

The non-dimensional velocities $(\mu, \lambda)$, which are parallel and vertical to the reference plane, can be derived as (7) and (8) [5].

(The reference plane is perpendicular to the control axes).

$$
\begin{aligned}
& \mu=\frac{V \cos \left(\alpha_{r}\right)}{R \Omega} \\
& \lambda=\frac{V \sin \left(\alpha_{r}\right)+v}{R \Omega}
\end{aligned}
$$

$\alpha_{r}$ is the reference plane angle of attack which, unlike the reference plane angle of attack in the helicopter, is above the longitudinal body axes in the forward flight [6].

For the rotor momentum, $T=\dot{m} \Delta V$ where $T$ is the main rotor thrust. $\dot{m}$ is the air mass rate crossing from the reference plane in the forward flight. From the reference [3], $\Delta V=2 v$ and $v$ is the main rotor induced velocity in the forward flight. Consider the air current velocity crossing from the reference plane is $V^{\prime}$ and the rotor Thrust coefficient is $C_{T}[7,8]$. Therefore

$$
\begin{aligned}
& T=\dot{m} \Delta V=\rho \pi R^{2} V^{\prime}(2 v) \Rightarrow v=\frac{T}{2 \rho \pi R^{2} V^{\prime}} \\
& V^{\prime}=\sqrt{\left(V \sin \left(\alpha_{r}\right)+v\right)^{2}+\left(V \cos \left(\alpha_{r}\right)\right)^{2}} \\
& \Rightarrow V^{\prime}=R \Omega\left(\sqrt{\lambda^{2}+\mu^{2}}\right) \\
& C_{T}=\frac{T}{\rho \pi R^{2}(R \Omega)^{2}} \\
& v=\frac{1}{2} \frac{\left(C_{T} R \Omega\right)}{\sqrt{\lambda^{2}+\mu^{2}}}
\end{aligned}
$$

The equation (9) is the blade momentum equation.

According to the Fig. (5) the air speed components in the blade section of $r$ can be derived. Consider $\Omega=\frac{d \psi}{d t}$ or $\psi=\int \Omega . d t$ and $\beta$ is the rotor flapping angle.

$u_{T}=\left(r \Omega+V \cos \left(\alpha_{r}\right) \sin (\psi)\right) \cos (\beta)$

$$
\begin{aligned}
& u_{P}=\left(V \sin \left(\alpha_{r}\right)+v\right) \cos (\beta)+r \frac{d \beta}{d t}+V \cos \left(\alpha_{r}\right) \cos (\psi) \sin (\beta) \\
& \phi=\tan ^{-1}\left(\frac{u_{P}}{u_{T}}\right) \\
& u=\sqrt{u_{P}{ }^{2}+u_{T}{ }^{2}}
\end{aligned}
$$

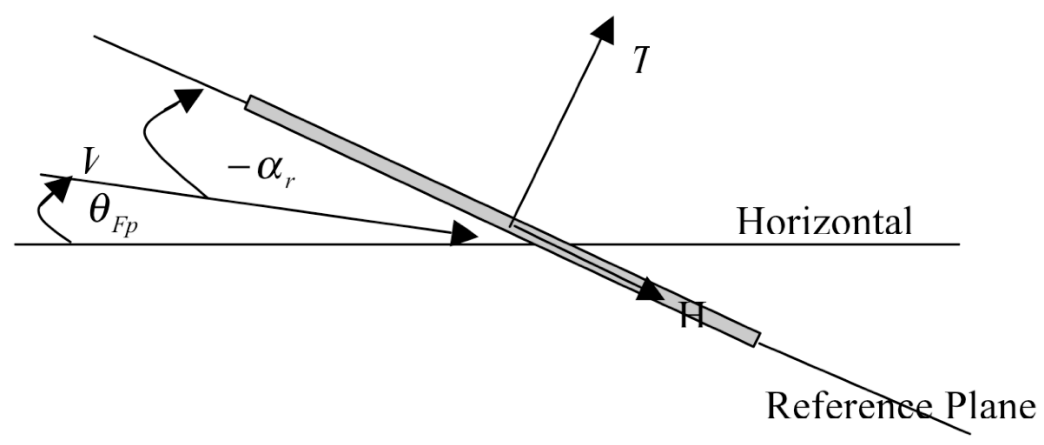

Fig. (4). Main rotor longitudinal forces. 
(a)

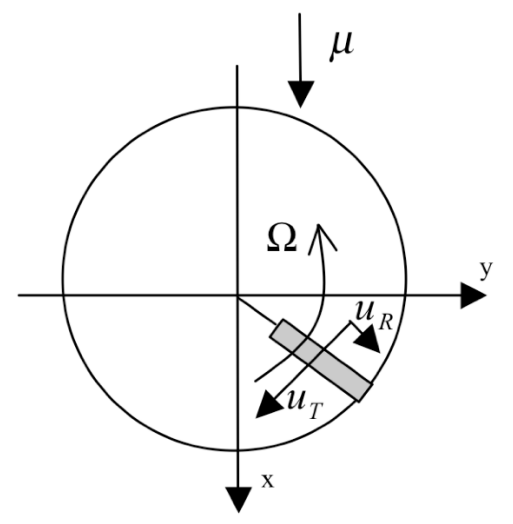

(b)

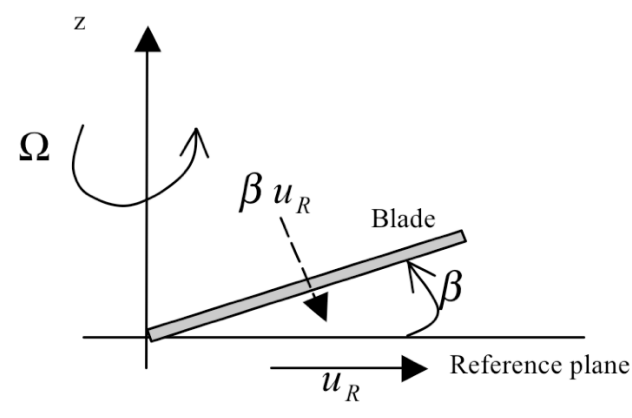

Fig. (5). Tangential and radial air speed components respect to the blade in the forward flight; (a) $u_{T}, u_{R}$ and (b) $u_{P}=\beta u_{R}$.

The gyroplane forces and moments coefficients are similar to them in the helicopter but its reference plane angle of attack and blade pitch angle will be negative respect to them in the helicopter. Therefore from the reference $[9,10]$, in the reference plane axes the rotor thrust and drag and side forces, $(T, H, Y)$, can be derived:

$$
\begin{aligned}
& T=C_{T} \rho \pi R^{2}(R \Omega)^{2} \\
& H=C_{H} \rho \pi R^{2}(R \Omega)^{2} \\
& Y=C_{Y} \rho \pi R^{2}(R \Omega)^{2}
\end{aligned}
$$

where $\left(C_{T}, C_{H}, C_{Y}\right)$ are thrust, drag and side force coefficients.
$F_{r}$ is radial force in the blade plane. $F_{x}$ is the force which is in the blade plane and $F_{z}$ is vertical force which is perpendicular to the blade plane.

The pitch and flapping angles respect to blade plane and

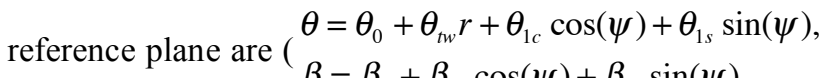
$\beta=\beta_{0}+\beta_{1 c} \cos (\psi)+\beta_{1 s} \sin (\psi)$ which are shown in the Figs. $(\mathbf{7}, \mathbf{8})$.

From the reference [10-12];

$\frac{C_{T}}{\sigma a}=\frac{1}{2 \pi} \int_{0}^{2 \pi} \int_{0}^{1} \frac{F_{z}}{a c} d r d \psi$

Lift and drag $(l, d)$ for a section of blade:
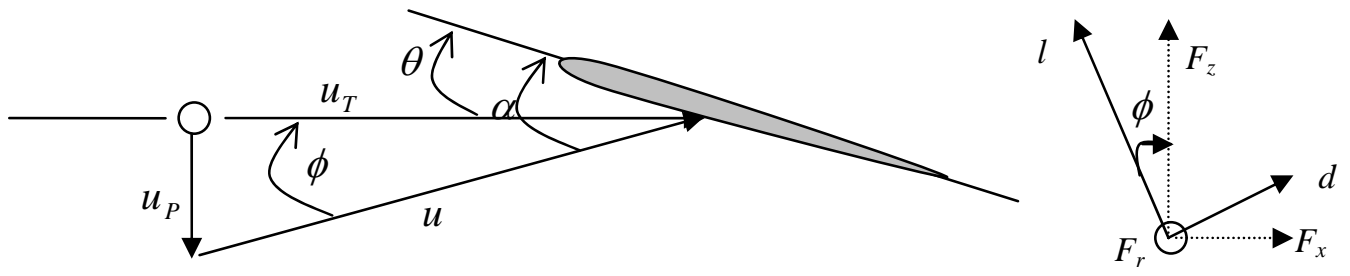

Fig. (6). Blade section aerodynamics and forces.

$l=\frac{1}{2} \rho u^{2} c c_{l}$

$d=\frac{1}{2} \rho u^{2} c c_{d}$

$c_{l}, c_{d}, c, u$, $\varnothing$ are lift, drag coefficient, cord, air speed and induced angle for each section of the blade. Therefore

$$
\begin{gathered}
F_{z}=l \cos (\phi)-d \sin (\phi) \\
F_{x}=l \sin (\phi)+d \cos (\phi) \\
F_{r}=-\beta F_{z}
\end{gathered}
$$

$F_{z}, F_{x}, F_{r}$ are the airfoil forces in each section of the blade and are perpendicular to each other.

$$
\begin{aligned}
& \frac{C_{H}}{\sigma a}=\frac{1}{2 \pi} \int_{0}^{2 \pi} \int_{0}^{1}\left(\frac{F_{x}}{a c} \sin (\psi)+\frac{F_{r}}{a c} \cos (\psi)\right) d r d \psi \\
& \frac{C_{Y}}{\sigma a}=\frac{1}{2 \pi} \int_{0}^{2 \pi} \int_{0}^{1}\left(-\frac{F_{x}}{a c} \cos (\psi)+\frac{F_{r}}{a c} \sin (\psi)\right) d r d \psi \\
& \sigma=\frac{N c R}{\pi R^{2}}, \gamma=\frac{\rho a c R^{4}}{I_{b}}, I_{b}=\frac{m}{3} R^{3}
\end{aligned}
$$

$N$ and $m$ are the number of blade and the mass for a blade. $I_{b}$ is inertial momentum about the flapping line for a blade and $a$ is the rotor lift coefficient [13]. After integrating

$$
C_{T}=\frac{\sigma a}{2}\left[\frac{\theta_{0}}{3}\left(1+\frac{3}{2} \mu^{2}\right)+\frac{\theta_{t w}}{4}\left(1+\mu^{2}\right)-\frac{\lambda}{2}\right]
$$




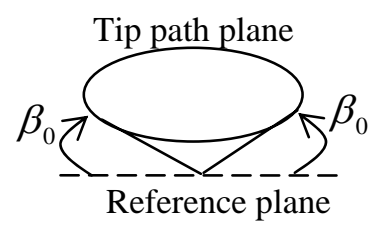

(a)

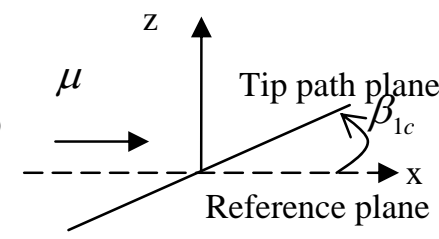

(b)

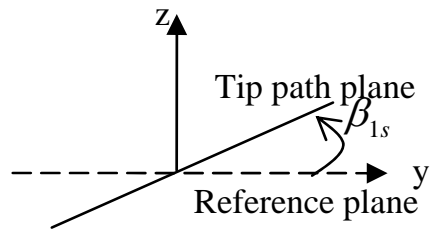

(c)

Fig. (7). (a); Flapping cone angle. (b); Longitudinal flapping angle (left view). (c); Lateral flapping angle (rear view).

(a)

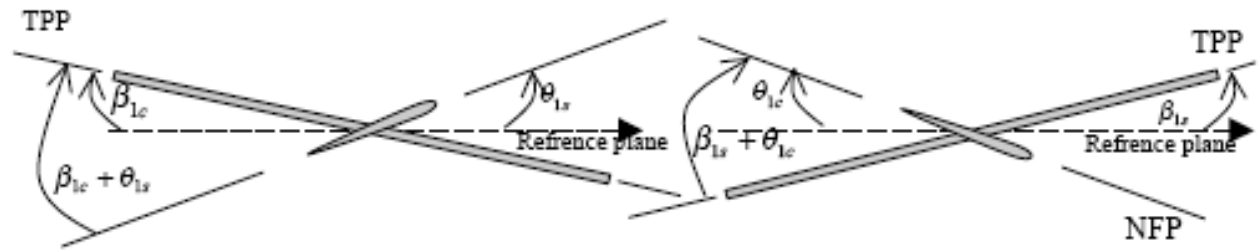

Fig. (8). (a); Tip Path Plane (TPP) longitudinal tilt respect to No Feathering Plane (NFP), (advance side view). (b); Tip Path Plane lateral tilt respect to No Feathering Plane (rear view). $\varnothing$ and $\beta$, the induced and flapping angles are small respectively. Therefore $\sin (\phi) \approx \phi, \quad \cos (\phi) \approx 1, \quad \sin (\beta) \approx \beta, \quad \cos (\beta) \approx 1, \quad-8<\beta<+8$

$C_{Y}=\frac{\sigma a}{2}\left[\theta_{0}\left(-\frac{1}{3} \beta_{1 c}+\frac{1}{2} \mu \lambda\right)+\theta_{t w}\left(-\frac{1}{4} \beta_{1 c}+\frac{1}{4} \mu \lambda\right)-\right.$

$\frac{1}{6} \theta_{1 c} \beta_{0}+\theta_{1 s}\left(-\frac{1}{4} \mu \beta_{1 c}+\frac{1}{4} \lambda\right)$

$\left.+\frac{3}{4} \lambda \beta_{1 c}+\frac{1}{6} \beta_{0} \beta_{1 s}+\frac{1}{4} \mu\left(\beta_{0}{ }^{2}+\beta_{1 c}{ }^{2}\right)\right]+\frac{\sigma c d_{0}}{4} \mu$

$C_{H}=-\frac{\sigma a}{2}\left[\theta_{0}\left(\frac{3}{2} \mu \beta_{0}+\frac{1}{3} \mu \beta_{1 s}\left(1+\frac{3}{2} \mu^{2}\right)\right.\right.$

$+\theta_{t w}\left(\frac{1}{2} \mu \beta_{0}+\frac{1}{4} \beta_{1 s}\left(1+\mu^{2}\right)\right)+\theta_{1 c}\left(\frac{1}{4} \lambda+\frac{1}{4} \mu \beta_{1 c}\right)$

$+\theta_{1 s}\left(\frac{1}{2} \mu \beta_{1 s}+\frac{1}{6} \beta_{0}\left(1+3 \mu^{2}\right)\right)-\frac{3}{4} \lambda \beta_{1 s}$

$+\beta_{0} \beta_{1 c}\left(\frac{1}{6}-\mu^{2}\right)-\frac{3}{2} \mu \lambda \beta_{0}-\frac{1}{4} \mu \beta_{1 s} \beta_{1 c}$

By attention to the Figs. $(7,8)$ suppose the blade pitch and flapping angles are

$\theta=\theta_{0}+\theta_{t w} r$

$\beta=\beta_{0}+\beta_{1 c} \cos (\psi)+\beta_{1 s} \sin (\psi)$

$\theta_{1 s}=\theta_{1 c}=0$

From the reference [13] the flapping angle can be derived as

$\beta_{0}=\gamma\left[\frac{\theta_{0}}{8}\left(1+\mu^{2}\right)+\frac{\theta_{t w}}{10}\left(1+\frac{5}{6} \mu^{2}\right)-\frac{\lambda}{6}\right]$

$\beta_{1 s}=\frac{(-4 / 3) \mu \beta_{0}}{1+\frac{1}{2} \mu^{2}}$

$\beta_{1 c}=2 \mu \frac{\left(\frac{4}{3} \theta_{0}+\theta_{t w}-\lambda\right)}{\frac{\mu^{2}}{2}-1}$
For each section of the gyroplane the forces should be derived [14-16]. Therefore

1- $\quad$ For the engine $Y_{P}=Z_{P}=i_{P}=0$ where $i_{P}$ is the engine incidence angle respect to the longitudinal body axes and $T_{P}$ is along the longitudinal body axes.

$X_{P}=T_{P}$ and $R_{P}=P_{P} / \omega$

Consider $\alpha_{b}=\theta_{b}-\theta_{f p}$, where $\alpha_{b}$ and $\theta_{f p}$ are the body angle of attack and flight path angle.

The horizontal tail, vertical tail and body forces in the wind axes should be converted to the body axes by the transformation $T F=T \alpha_{b} \quad T \beta_{b}$ where;

$$
\begin{aligned}
& T \beta_{b}=\left(\begin{array}{ccc}
\cos \left(\beta_{b}\right) & -\sin \left(\beta_{b}\right) & 0 \\
\sin \left(\beta_{b}\right) & \cos \left(\beta_{b}\right) & 0 \\
0 & 0 & 1
\end{array}\right), \\
& T \alpha_{b}=\left(\begin{array}{ccc}
\cos \left(\alpha_{b}\right) & 0 & -\sin \left(\alpha_{b}\right) \\
0 & 1 & 0 \\
\sin \left(\alpha_{b}\right) & 0 & \cos \left(\alpha_{b}\right)
\end{array}\right)
\end{aligned}
$$

2- $\quad$ The main rotor forces in the body axes;

The main rotor forces in the wind axes should be converted to the body axes by the transformation $T F m=T m T \beta_{b}$ where;

$$
\operatorname{Tm}=\left(\begin{array}{ccc}
\cos \left(\alpha_{r}\right) & 0 & -\sin \left(\alpha_{r}\right) \\
0 & 1 & 0 \\
\sin \left(\alpha_{r}\right) & 0 & \cos \left(\alpha_{r}\right)
\end{array}\right)
$$

Therefore the main rotor forces in the body axes will be as $F m=T F m \cdot(H, Y, T)^{T}$, (the superscript $T$ represents transposition). 


$$
\left(\begin{array}{l}
X_{M} \\
Y_{M} \\
Z_{M}
\end{array}\right)=\left(\begin{array}{c}
-F m(1) \\
F m(2) \\
-F m(3)
\end{array}\right)
$$

$F m(i)$ represents the $i^{\text {th }}$ element of the $F m$ vector, $\mathrm{i}=1,2,3$.

Consider $C_{d}, C_{L}, C_{d 0}, C_{L 0}$, and $C_{L \alpha}$, represent drag, lift coefficient, drag coefficient for the zero angle of attack, lift coefficient for the zero angle of attack and lift coefficient derivative respect to angle of attack.

$L$ and $D$ represent aerodynamics surfaces lift and drag in the wind axes.

3- The horizontal tail forces;

$F h=T F .\left(D_{H}, 0, L_{H}\right)^{T}$

Consider the interference angle on the horizontal tail by the engine, main rotor, fuselage and flight path be zero. Therefore $\alpha_{H}=\alpha_{b}$ and the horizontal tail forces in the body axes will be as

$$
\left(\begin{array}{c}
X_{H} \\
Y_{H} \\
Z_{H}
\end{array}\right)=\left(\begin{array}{c}
-F h(1) \\
F h(2) \\
-F h(3)
\end{array}\right)
$$

where $F h(\mathrm{i})$ represents the $\mathrm{i}^{\text {th }}$ element of the $F h$ vector, $\mathrm{i}=1,2,3$.

$$
\begin{aligned}
& L_{H}=\left(\eta_{H}\right) q A_{H} C_{L_{H}} \\
& D_{H}=\left(\eta_{H}\right) q A_{H}\left[\frac{\left(C_{L}\right)^{2}}{\pi \cdot A R}\left(1+\delta_{i}\right)+C_{d_{0}}\right]_{H} \\
& C_{L_{H}}=\left(C_{L_{0}}+C_{L_{\alpha}} \alpha\right)_{H} \\
& \eta_{H}=q_{H} / q, q=(1 / 2) \rho u^{2} .
\end{aligned}
$$

$A_{H}, A R_{H}$ and $q_{H}$ are area, aspect ratio and dynamics pressure of the horizontal tail.

$\delta_{\mathrm{i}}$ is tail span efficiency coefficient. Suppose $\left(C_{L 0}\right)_{H}=$ 0 .

4- Vertical tail forces:

$F v=T F .\left(D_{V}, L_{V}, 0\right)^{T}$

Consider interference angle on the vertical tail by the engine, main rotor and flight path is zero. Therefore $\beta_{V}=\beta_{b}$ and the vertical tail forces in the body axes will be as

$$
\left(\begin{array}{c}
X_{V} \\
Y_{V} \\
Z_{V}
\end{array}\right)=\left(\begin{array}{c}
-F v(1) \\
F v(2) \\
-F v(3)
\end{array}\right)
$$

$F v$ (i) represents the $\mathrm{i}^{\text {th }}$ element of the $F v$ vector, $\mathrm{i}=1,2,3$.

Consider $C_{L \beta}$ represent vertical tail lift coefficient derivative respect to side slip angle. Therefore
$L_{V}=\left(\eta_{V}\right) q A_{V} C_{L_{V}}$

$D_{V}=\left(\eta_{V}\right) q A_{V}\left[\frac{\left(C_{L}\right)^{2}}{\pi \cdot A R}\left(1+\delta_{i}\right)+C_{d_{0}}\right]_{V}+\Delta D_{i n}$

$C_{L_{V}}=\left(C_{L_{0}}+C_{L_{\beta}} \beta+C_{L_{\delta R}} \delta R\right)_{V}$

$\eta_{V}=q_{V} / q$.

$A_{V}, A R_{V}$ and $q_{V}$ are area, aspect ratio and dynamics pressure of the vertical tail.

$\Delta D_{\text {in }}$ is vertical tail interference drag and $\delta_{i}$ is tail span efficiency coefficient. Suppose $\left(C_{L 0}\right)_{V}=0$ and $\Delta D_{i n}=$ 0 .

5- Fuselage forces;

$F f=T F .\left(D_{F}, S F_{F}, L_{F}\right)^{T}$

where $S F_{F}$ is the fuselage side force.

Consider interference angle on the fuselage by the main rotor and flight path is zero. The vertical tail forces in the body axes will be as;

$\left(\begin{array}{c}X_{F} \\ Y_{F} \\ Z_{F}\end{array}\right)=\left(\begin{array}{c}-F f(1) \\ F f(2) \\ -F f(3)\end{array}\right)$

where $F f(i)$ represents the $i^{\text {th }}$ element of the $F f$ vector, $\mathrm{i}=1,2,3$.

$D_{F}=C_{d_{F}} q A_{F}$

$Y_{F}=C_{Y_{F}} q A_{F}$

$L_{F}=C_{L_{F}} q A_{F}$

$C_{Y_{F}}=\left(C_{Y_{0}}+C_{Y_{\beta}} \beta\right)_{F}$

$C_{L_{F}}=\left(C_{L_{0}}+C_{L_{\alpha}} \alpha\right)_{F}$

$A_{F}$ is the gyroplane wetted area. consider $C_{d_{F}}$ be constant.

$C_{Y_{\beta}}$ is side force coefficient derivative respect to side slip angle.

For the energy balance equation, all consumed powers are equal to all produced powers. Therefore; $P=\frac{d W_{t}}{d t}=\mathbf{F} . \mathbf{V}+\mathbf{M} \cdot \dot{\Theta}$

where $P, d W_{t}$, represent power and total work differential. F, V, $\mathbf{M}$ and $\boldsymbol{\Theta}$ are force, velocity, momentum and angular velocity vectors. According to the trim condition $\dot{\boldsymbol{\Theta}}=0$ therefore $P=\frac{d W_{t}}{d t}=\mathbf{F} . \mathbf{V}$.

The consumed work is equal to the air displacement respect to body to the force which is merged form the displacement. 
The efficient work is equal to the body displacement respect to the inertial system to the force.

Therefore the powers can be written as:

$$
\begin{aligned}
& P_{i}=T V, P_{p r o f}=H V \cos \left(\alpha_{r}\right), \\
& P_{p a r a z}=D_{B H V} V, P_{c}=W V \sin \left(\theta_{F P}\right)
\end{aligned}
$$

The energy balance equation will be as (13)

$$
\begin{aligned}
& H V \cos \left(\alpha_{r}\right)+D_{B H V} V+W V \sin \left(\theta_{F P}\right)+ \\
& T V=T_{P} V \cos \left(\alpha_{b}\right) \cos \left(\beta_{b}\right)+P_{M_{\text {toral }}}-P_{f M}
\end{aligned}
$$

$W$ is the gyroplane weight. $D_{B H V}$ is the gyroplane body, horizontal tail and vertical tail drag. $P_{M_{\text {towat }}}$ represents the main rotor engine power which is zero. Consider $\theta_{F P}=0$ and $P_{M_{\text {tootal }}}=0$.

\section{AUTOROTATION MECHANISM}

The air current has two components respect to the blade plane. One component is the perpendicular component. It is small near the hub because the induced velocity is high in there. Another component is in the blade plane which increases from root to tip. For this reason the induced angle increases near the root therefore the blade angle of attack increases and the stall occurs in there. In the tip and root region, the induced drag is smaller than the profile drag. These two regions take air current energy. In the middle region (between tip and root region) the induced drag conquers on the profile drag and produces rotating torque by using air current energy. Its torque is more than opposite torque in the tip and root regions. This is shown in the Fig. (10). The stall region and produced and consumed power regions of the rotor are shown obviously in the Fig. (10) [1719].

The rotating torque $(d Q)$ for the blade section is

$$
\begin{aligned}
& d Q=(d-\phi l) y d y=0 \\
& \Rightarrow \frac{c_{d}}{c_{l}}=\phi=\alpha-\theta
\end{aligned}
$$

$\theta$ is the blade pitch angle respect to blade plane and $\alpha$ is the angle of attack for the blade section respect to $u$.

$y$ is the blade section position from hub.

According to the auto rotation diagram (Fig. 11), there is three regions: 1- near the root $c_{d} / c_{l}$ is small and the produced torque conquers on the consumed torque. Therefore it tends to increase the rotor rpm. 2- Near the tip $c_{d} / c_{l}$ is large and the consumed torque conquers on produce torque. Therefore it tends to decreases the rotor rpm. 3- When $c_{d} / c_{l}$ becomes equal to induced angle the consumed torque will be equal to the produced torque. Therefore for each pitch angle the trim condition of the blade element will be occurred on the line of 45 degrees. By attention to the point $\mathbf{A}$ in the Fig. (11), the pilot should uses air current energy optimally. In order to reach this goal the pilot should adjust the blade's pitch angle so that the induced angle line of 45 degrees interacts the diagram of $c_{d} / c_{l} \leftrightarrow \alpha$ in the minimum point [20-22].

For the gyroplane, model JT-5B, the trim equations for the several flight scenarios will be solved. The units of all parameters are metric. Consider $\theta_{b}=0, \theta_{F p}=0$ and $\beta_{b}=-0.01$ $(\mathrm{rad})$. The flight is done in sea level therefore $\rho=1.23$. The results will be as which is shown in Table $\mathbf{1}$.

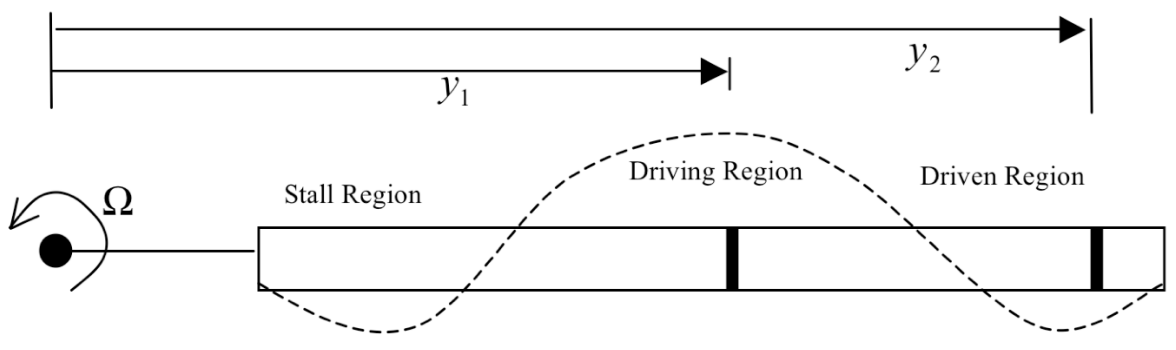

Net positive in plane force (Produces power to rotor)

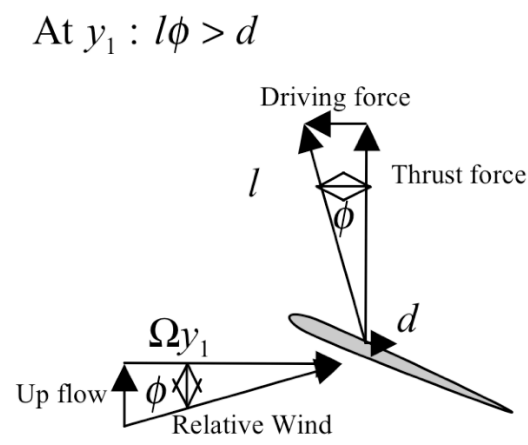

Net negative in plane force (Consumes power)

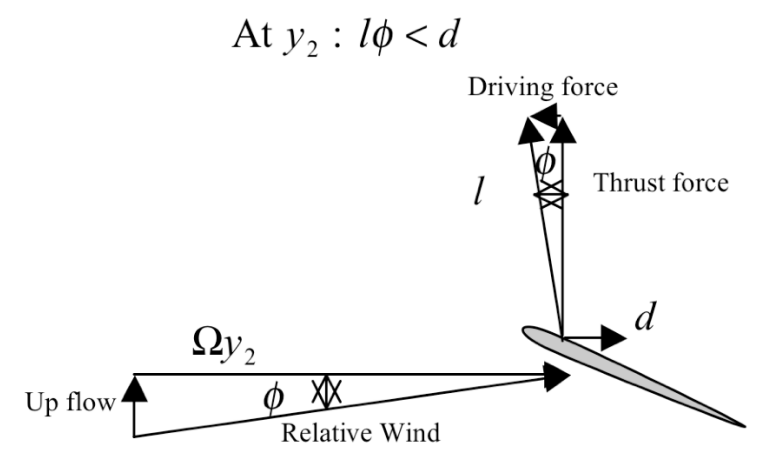

Fig. (9). Air current consumed and air current produced rotating power for the different sections of the blades in the vertical autorotation. 


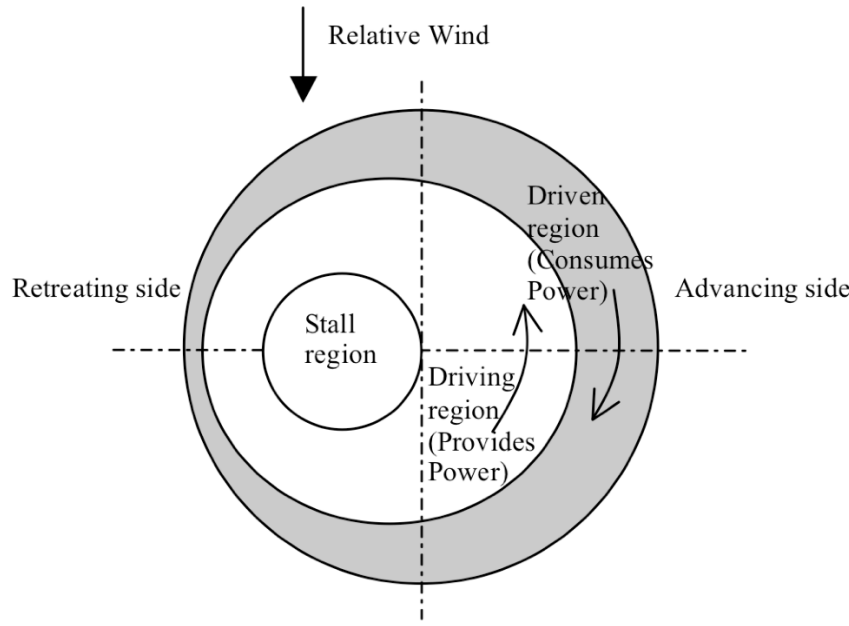

Fig. (10). Distributed autorotation power on the rotor plane in the forward flight.

The mathematical software is used for this problem. Newton's method for the find root command converged the equations with maximum iteration 5000, accuracy goal 10 and working precision 10 .

Maxiteration specify the maximum number of steps to take in trying to find a solution.

AccuracyGoal is an option for various numerical operations which specifies how many digits of accuracy should be sought in the final result. AccuracyGoal effectively specifies the absolute error allowed in a numerical procedure.

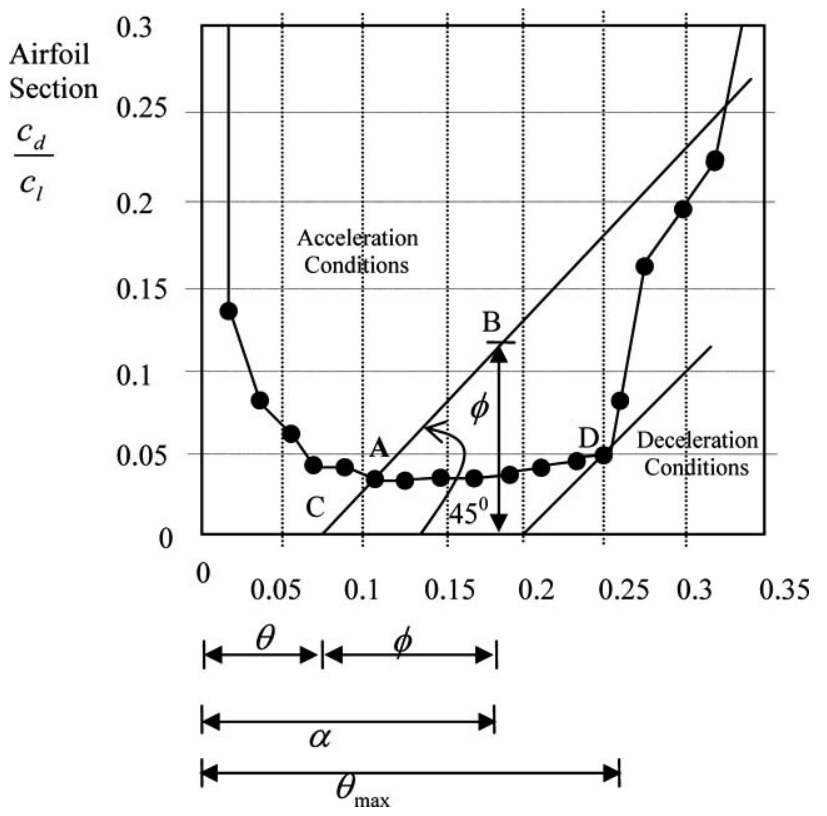

Fig. (11). autorotation diagram for describing trim condition in the blade element.

WorkingPrecision is an option for various numerical operations which specifies how many digits of precision should be maintained in internal computations. Setting WorkingPrecision- $>n$ causes all internal computations to be done to at most $n$-digit precision.
Table 1. Gyroplane Trim Parameters for Three Speed and Three Weight Scenarios

\begin{tabular}{|c|c|c|c|c|}
\hline \multicolumn{5}{|c|}{$V=19(\mathrm{~m} / \mathrm{s})$} \\
\hline$W(N)$ & $\alpha_{r}(\mathrm{rad})$ & $\theta_{0}(\mathrm{rad})$ & $\delta_{r}(\mathrm{rad})$ & $T_{P}(N)$ \\
\hline 2000 & -0.0588 & -0.01123 & 0.0119673 & 820.643 \\
\hline 2400 & -0.0707 & -0.0137 & 0.01208 & 879.009 \\
\hline 2800 & -0.0826 & -0.0151 & 0.0121 & 946.8 \\
\hline \multicolumn{5}{|c|}{$V=22(\mathrm{~m} / \mathrm{s})$} \\
\hline$W(N)$ & $\alpha_{r}(r a d)$ & $\theta_{0}(\mathrm{rad})$ & $\delta_{r}(\mathrm{rad})$ & $T_{P}(N)$ \\
\hline 2300 & -0.05037 & -0.01089 & 0.01198 & 1054.31 \\
\hline 2350 & -0.0514 & -0.01125 & 0.01199 & 1060 \\
\hline 2450 & -0.0536 & -0.01191 & 0.01202 & 1072.46 \\
\hline \multicolumn{5}{|c|}{$V=25(m / s)$} \\
\hline$W(N)$ & $\alpha_{r}(r a d)$ & $\theta_{0}(\mathrm{rad})$ & $\delta_{r}(r a d)$ & $T_{P}(N)$ \\
\hline 2350 & -0.0397 & -0.0086 & 0.01193 & 1296.95 \\
\hline 2400 & -0.04065 & -0.0091 & 0.01194 & 1301.81 \\
\hline 2500 & -0.0423 & -0.0099 & 0.01197 & 1311.83 \\
\hline
\end{tabular}

According to Table $\mathbf{1}$ the results are reasonable because the gyroplane reference plane angle of attack is obtained negative. This means unlike the helicopter reference plane the gyroplane reference plane has the positive angle of attack respect to the longitudinal body axes. Therefore it takes up wash for auto rotating and produces drag and sufficient lift. The pilot pitch angle for the blade is obtained negative. The gyroplane rotor has not initial $\mathrm{rpm}$ as helicopter rotor. Therefore its blades should take negative pitch angle to do autorotation counter clockwise as the helicopter rotor [23, 24].

\section{CONCLUSION}

Extraction and solution gyroplane trim equations shows that for the various flight conditions and parameters how much the pilot should change the trim control devices. It also shows that "are these flight conditions possible in addition to the limitation of trim control devices?" From the results, the answers of the many requirement questions will be obvious in the gyroplane design cycle and the gyroplane design cycle converges too fast. Then the size of its different parts can be revised. The more accurate data gives the more accurate results. Therefore it is suggested for obtaining the more accurate data; the wind tunnel tests with real size should be used [25].

\section{NOMENCLATURE}

$\begin{array}{ll}T_{P} & =\text { Propeller Thrust } \\ V & =\text { Gyroplane forward velocity } \\ W & =\text { Gyroplane gravity weight } \\ \alpha_{r} & =\text { Reference plane angle of attack }\end{array}$


$\theta_{0} \quad=\quad$ Pilot blade pitch angle

$\delta_{r} \quad=\quad$ Rudder angle

\section{REFERENCES}

[1] J. Cierva, and D. Rose. Wings of Tomorrow: The Story of the Autogiro. NY: Brewer Warren \& Putnam, 1931.

[2] R.B. Lewis, "Army helicopter performance trends", J. Am. Helicopter Soc., vol. 17, no. 2, pp. 15, 23, 1972.

[3] R.W. Prouty, Helicopter Performance, Stability and Control, Boston MA: PWS Engineering Publishing, 1986, pp. 177, 187.

[4] S. Newman, The Foundation of Helicopter Flight. London: Edward Arnold, 1994, pp. 122-127.

[5] J.B. Wheatley, The Aerodynamics Analysis of the Gyroplane Rotating Wing System, National Advisory Committee: Technical Note, No. 492-1933.

[6] A. Gessow, and G.C. Myers, Aerodynamics of the Helicopter, Macmilam Co., 1952, New York: Frederick Ungar Publishing, 1967, p.121.

[7] W. Z. Stepniewski, Basic aerodynamics and performance of the helicopter, AGARD Lecture Series LS-63, 1973.

[8] W.Z. Stepniewski, and C.N. Key, Rotary Wing Aerodynamics. Part 2, Chapters 2 \& 3, New York: Dover Publication, 1984.

[9] B.W. McCormick, Aerodynamics, Aeronautics and Flight Mechanics, Chapter 7. New York: John Wiley \& Sons, Inc, 1995.

[10] G.L. Leishman, Principal of Helicopter Aerodynamics. Chapters 4 \& 5, Cambridge: Cambridge University Press, 2000.

[11] F.D. Harris, "Articulated rotor blade flapping motion at low advance ratio", J. Am. Helicopter Soc., vol. 17, no. 1, pp. 41-48, 1972.

[12] P.P. Friedman, "Recent development in rotary-wing aeroelasticity", J. Aircraft, vol. 14, no. 11, pp. 1027-1041, 1977.
[13] W. Johnson, Helicopter Theory, Chapter 5. Princeton, NJ: Princeton University Press, 1980.

[14] C.N.H. Lock, Further Development of Autogiro Theory, Aeronautical Research Council, Research Reports and Memoranda $1127,1927$.

[15] H. Glauert, On the horizontal flight of a helicopter, Aeronautical Research Council, Research Reports and Memoranda 1157, 1928.

[16] H. Glauert, On the horizontal flight of a helicopter, ARCR\& M1730, 1928.

[17] J.B. Wheatley, Lift and drag characteristics and gliding performance of an autogiro as determined in flight. NACA Report No. 434, 1932.

[18] G.T. White, A.H. Logan, and J.D. Graves, An Evaluation of Helicopter Autorotation Assist Concept., 38th Annual Forum of the American Helicopter Soc, Anaheim, CA, 4-7 May, 1982.

[19] E.A. Fradenburgh, "A simple autorotational flare index", J. Am. Helicopter Soc., vol. 29, no. 3, pp. 73-74,1984.

[20] F.B. Gustafson, and G.C. Myers, Stalling of Helicopter Blades., NACA Rep. No. 840, 1946.

[21] R.G. Loewy, "Review of rotary wing V/STOL dynamics aeroelastic problems", J. Am. Helicopter Soc, vol. 14, no. 3, pp. 324, 1969.

[22] T.L. Wood, High energy rotor system, 32th Annual V/STOL Forum of the American Helicopter Society, Washington Dc, 10-12 May, 1976.

[23] Q. Wald, "A method for rapid stimation of helicopter performance", J. Aeronaut. Sci., vol. 10, no. 4, pp. 131-135, 1943.

[24] A. Gessow, and G.C. Myers, Flight tests of a Helicopter in autorotation, including a comparison with theory. NACA TN 1267, 1947.

[25] A.F. Lehman, and J.A. Besold. Tests section size influence on model helicopter rotor performance, USAAVLABS TR 71-6, 1971.

(C) Osaji and Farrokhfal; Licensee Bentham Open.

This is an open access article licensed under the terms of the Creative Commons Attribution Non-Commercial License (http: //creativecommons.org/licenses/by$\mathrm{nc} / 3.0 /$ ) which permits unrestricted, non-commercial use, distribution and reproduction in any medium, provided the work is properly cited. 